\title{
Random Ising model in three dimensions: theory, experiment and simulation - a difficult coexistence
}

\author{
B.Berche*1, P.E.Berche ${ }^{\dagger 2}$, C.Chatelain ${ }^{\ddagger 1}$, W.Janke . $^{\S 3}$ \\ 1 Groupe M, Laboratoire de Physique des Matériaux, \\ UMR CNRS 7556, \\ Université Henri Poincaré, Nancy 1 , \\ F-54506 Vandœuvre les Nancy Cedex, France \\ 2 Groupe de Physique des Matériaux, UMR CNRS 6634, \\ Université de Rouen, \\ F-76801 Saint Etienne du Rouvray Cedex, France \\ 3 Institut für Theoretische Physik, \\ Universität Leipzig, \\ D-04109 Leipzig, Germany
}

Received November 11, 2004

We discuss different approaches to the study of the effect of disorder in the three-dimensional Ising model. From the theoretical point of view, renormalization group calculations provide quite accurate results. Experiments carried out on crystalline mixtures of compounds lead to measurements as accurate as three digits on the values of critical exponents. Numerically, extensive Monte Carlo simulations then pretend to be of comparable accuracy. Life becomes complicated when details are compared between the three approaches.

Key words: random Ising model, renormalization group, Monte Carlo simulations, effective critical exponents

PACS: $05.40 .+j, 64.60 . F r, 75.10 . H k$

\section{Introduction}

For many years the random Ising model has served as a paradigmatic system in which the effect of disorder may be studied through different techniques. Here we

\footnotetext{
${ }^{*}$ E-mail: berche@lpm.u-nancy.fr

${ }^{\dagger}$ E-mail: pierre.berche@univ-rouen.fr

${ }^{\ddagger}$ E-mail: chatelai@lpm.u-nancy.fr

$\S$ E-mail: wolfhard.janke@itp.uni-leipzig.de
} 
now mention three of them. The renormalization group (RG) approach, experimental measurement and Monte Carlo simulation.

RG calculations were considered quite early $[1,2]$ and since then many groups were illuminated by skillful RG calculations. Among them, we would like to mention the work of Folk, Holovatch and Yavors'kii (for recent reviews, see [3,4]). On the experimental side, measurements on crystalline mixtures of difluoride of different transition metals, e.g., magnetic $\mathrm{FeF}_{2}$ substituted with non-magnetic $\mathrm{ZnF}_{2}$, were performed in the same period over two decades, gaining in refinement and accuracy (see early results of Birgeneau et al. in [5], for a review see, e.g., [3]). For the third aspect of simulations, technical progress made by computer manufacturers urged more and more accurate simulations (which started for disordered systems in $3 D$, e.g., with Landau in [6]) and the study of disordered magnetic systems benefited from the development of parallel computing. Monte Carlo simulators thus competed in performance (for a review, see, e.g., [3]).

To introduce the subject, we may imagine a conversation between three people a few decades ago, when the concept of universality was not as clearly stated as it is nowadays. Imagine a theoretician as the one who stands up with enthusiasm for this new concept. Let us call him Salviati. He has an interesting discussion with a good physicist, an experimentalist, let us say named Sagredo. It is usually considered that the concept of "numerical experiments" originates in the FPU problem, a numerical study of the thermalization of a chain of atoms, performed by Fermi, Pasta and Ulam at Los Alamos [7]. A third person is thus participating in our conversation. Having no more character free, let us call him Simplicio - the simulator!

In a pub, early in the seventies:

SAGR. Dear friends, I would like to report on recent experiments that I am conducting at the lab. I produced many samples of difluoride of magnetic transition metals, substituting randomly different amounts of non-magnetic metal, and found very interesting results.

SALV. It would be great to compare your results with recent theoretical predictions. Which quantity do you measure?

SAGR. Critical temperature, correlation length, susceptibility, ... Of course, the transition temperature decreases when impurities are added, but what looks interesting is the neighbourhood of the transition. The singularities of some quantities (susceptibility, correlation length) seem to be independent of the impurity concentration.

SALV. This is a wonderful observation. It supports the universality assumption. You know, from recent $R G$ theory, one expects that the free energy density has a singularity in the vicinity of the transition, and that this singularity is described by some critical exponents which are believed to be independent of the details of the system under consideration. 
SAGR. Do you mean that the presence of impurities is a detail? Experimentally it is not. It produces an observable decrease of transition temperature.

SALV. You are right, critical temperature is not universal and disorder is perhaps not a detail, but the precise amount of disorder probably does not matter, at least in some range.

SAGR. But the singularities that I measured are different from those of a pure sample that I have also produced. A colleague of mine made similar experiments on Heisenberg-like samples and he did not notice any similar modification of the singularities due to the introduction of disorder.

SALV. Probably that disorder is relevant in your case and not in his case.

Simpl. Maybe we could make Monte Carlo simulations, I have access to a computer and I have been told that it is not very difficult to produce simulations of a disordered Ising model on a cubic lattice. I only have to add quenched vacancies in the system and average over the disorder realizations.

SALV. We may also write an effective $\varphi^{4}$-theory for the diluted problem with a scalar field Ginzburg-Landau-Wilson Hamiltonian and calculate the critical exponents analytically.

SAGR. I will measure many other quantities and see what is universal and what is not, and we will compare our results.

And this is where the problems occur. In the comparison...

In the rest of the paper we will give a short review of some recent progress in the studies of the $3 D$ disordered Ising model, emphasising the role of universality and its difficult emergence when trying to reconcile theoretical, experimental, and computational predictions. Reference will be made to seminal papers and to exhaustive reviews only. It is of course easy to wander a bit on arXiv and look around the names of Calabrese, Pelissetto and Vicari, Prudnikov, Shalaev or Sokolov, Folk or Holovatch. We apologise to those whose work is not directly mentioned in the short reference list, an indelicacy only due to our ignorance, our misunderstanding or our laziness - or all together.

\section{RG calculation of critical exponents}

Long distance properties of the Ising model near its second-order phase transition are described in field theory by an effective Ginzburg-Landau-Wilson Hamiltonian

$$
H_{\text {Ising }}[\varphi]=\int \mathrm{d}^{D} \mathbf{r}\left[\frac{1}{2}(\boldsymbol{\nabla} \varphi(\mathbf{r}))^{2}+\frac{1}{2} m_{0}^{2} \varphi^{2}(\mathbf{r})+\frac{\tilde{u}_{0}}{4 !} \varphi^{4}(\mathbf{r})\right]
$$

where $m_{0}^{2}$ is the bare coupling proportional to the deviation $T-T_{\mathrm{c}}$ from the critical point and $\varphi(\mathbf{r})$ is a bare scalar field. Quenched randomness is introduced in such 
a model by considering that the adjunction of disorder results in a distribution of local transition temperatures, so that a random temperature-like variable $\Delta$ is simply added to $m_{0}^{2}$,

$$
H[\varphi, \Delta]=\int \mathrm{d}^{D} \mathbf{r}\left[\frac{1}{2}(\boldsymbol{\nabla} \varphi(\mathbf{r}))^{2}+\frac{1}{2}\left(m_{0}^{2}+\Delta\right) \varphi^{2}(\mathbf{r})+\frac{\tilde{u}_{0}}{4 !} \varphi^{4}(\mathbf{r})\right],
$$

where $\Delta$ is drawn from, e.g., a Gaussian probability distribution of zero mean and dispersion $\sigma^{2}, \mathcal{P}(\Delta)=\left(2 \pi \sigma^{2}\right)^{-1} \exp \left(-\Delta^{2} / 2 \sigma^{2}\right)$. For a specific disorder realization $[\Delta]$, the partition function and the free energy read as $Z[\Delta]=\int \mathcal{D}[\varphi] \mathrm{e}^{-\beta H[\varphi, \Delta]}$ and $F[\Delta]=-\beta^{-1} \ln Z[\Delta]$. The average over quenched disorder then requires to calculate quantities like $\overline{F[\Delta]}=-\beta^{-1} \int \mathcal{D}[\Delta] \ln Z[\Delta] \mathcal{P}(\Delta)$. This is performed through the introduction of $n$ replicas of the model (labelled by $\alpha$ ). Averaging over quenched disorder one ends up with an effective Hamiltonian with cubic anisotropy where the replicas are coupled through a new parameter $v_{0}$

$H_{\text {replicas }}[\varphi]=\int \mathrm{d}^{D} \mathbf{r}\left[\frac{1}{2} \sum_{\alpha=1}^{n}\left[\left(\boldsymbol{\nabla} \varphi_{\alpha}(\mathbf{r})\right)^{2}+m_{0}^{2} \varphi_{\alpha}^{2}(\mathbf{r})\right]+\frac{u_{0}}{4 !} \sum_{\alpha=1}^{n} \varphi_{\alpha}^{4}(\mathbf{r})+\frac{v_{0}}{4 !}\left(\sum_{\alpha=1}^{n} \varphi_{\alpha}^{2}(\mathbf{r})\right)^{2}\right]$

Here the bare coupling $u_{0}$, proportional to $\tilde{u}_{0}$, is positive and the bare coupling $v_{0}$, proportional to $-\sigma^{2}$, is negative. The properties of the random Ising model are recovered while taking the limit $n \rightarrow 0, \ln Z=\lim _{n \rightarrow 0}\left(Z^{n}-1\right) / n$. Under a change of length scale by a factor $\mu$, the field and couplings are renormalized according to

$$
\varphi=Z_{\phi}^{1 / 2} \phi, \quad m_{0}^{2}=Z_{m^{2}} m^{2}, \quad u_{0}=\mu^{\epsilon} \frac{Z_{u}}{Z_{\phi}^{2}} u, \quad v_{0}=\mu^{\epsilon} \frac{Z_{v}}{Z_{\phi}^{2}} v
$$

where $\epsilon=4-D$. The RG functions are defined by differentiation at fixed bare parameters,

$$
\begin{array}{ll}
\beta_{u}(u, v)=\left.\frac{\partial u}{\partial \ln \mu}\right|_{0}, & \beta_{v}(u, v)=\left.\frac{\partial v}{\partial \ln \mu}\right|_{0}, \\
\gamma_{\phi}(u, v)=\left.\frac{\partial \ln Z_{\phi}}{\partial \ln \mu}\right|_{0}, & \gamma_{m^{2}}(u, v)=\left.\frac{\partial \ln Z_{m^{2}}}{\partial \ln \mu}\right|_{0} .
\end{array}
$$

The skill of the theoretician is measured as his ability to compute these functions perturbatively, disentangling Feynman loops (they are known up to 6 loops), removing divergences which occur in the asymptotic limit by controlled rearrangement of the series for the vertex functions. Eventually expecting reliable results after complicated resummation procedures [8]. Fixed points are then solutions of $\beta_{u}\left(u^{*}, v^{*}\right)=\beta_{v}\left(u^{*}, v^{*}\right)=0$, the stability of which is controlled by a stability matrix $\partial \beta_{i} / \partial u_{j}$ with eigenvalues which besides the standard critical exponents also govern the corrections to scaling (exponent $\omega$ ).

At that point, even Simplicio may read off the critical exponents! Consider for example the pair correlation function of bare fields $\langle\varphi(0) \varphi(\mathbf{r})\rangle$. Under a change of 
length scale $\mu$, it renormalizes to $Z_{\phi}(\mu)\langle\phi(0) \phi(\mathbf{r})\rangle$. In the same manner, for another dilatation parameter, $\mu s$, one has $\langle\varphi(0) \varphi(s \mathbf{r})\rangle \rightarrow Z_{\phi}(\mu s)\langle\phi(0) \phi(s \mathbf{r})\rangle$. The ratio from this latter to the previous expression leads to

$$
\frac{\langle\phi(0) \phi(s \mathbf{r})\rangle}{\langle\phi(0) \phi(\mathbf{r})\rangle}=\frac{Z_{\phi}(\mu)}{Z_{\phi}(\mu s)} \frac{\langle\varphi(0) \varphi(s \mathbf{r})\rangle}{\langle\varphi(0) \varphi(\mathbf{r})\rangle} .
$$

This expression gives the algebraic decay of the two-point correlation function of renormalized fields $\langle\phi(0) \phi(\mathbf{r})\rangle \sim|\mathbf{r}|^{-\left(D-2+\eta_{\phi}\right)}$ in terms of the pair correlation function of the bare fields which are described by mean-field theory (MFT), i.e., at the Gaussian fixed point $(\mathrm{FP}),\langle\varphi(0) \varphi(\mathbf{r})\rangle \sim|\mathbf{r}|^{-(D-2)}\left(\eta_{\mathrm{MFT}}=0\right)$. The ratio $Z_{\phi}(\mu) / Z_{\phi}(\mu s)=\mathrm{e}^{\int_{\mu s}^{\mu} \gamma_{\phi} \mathrm{d} \ln \mu}$ evaluated at the new FP gives $s^{-\gamma_{\phi}^{*}}$ and leads to

$$
\frac{\langle\phi(0) \phi(s \mathbf{r})\rangle}{\langle\phi(0) \phi(\mathbf{r})\rangle} \sim s^{-\left(D-2+\gamma_{\phi}^{*}\right)},
$$

from which one reads off the value of the critical exponent at this FP:

$$
\eta_{\phi}=\gamma_{\phi}^{*}
$$

Following the same argument, the scaling dimension $1 / \nu$ of the (renormalized) temperature field $m^{2}$ is given at the random fixed point in terms of the MFT value, $1 / \nu_{\mathrm{MFT}}=2$, and one gets

$$
\frac{1}{\nu}=2-\gamma_{m^{2}}^{*}
$$

From these two exponents, the others may be deduced by scaling arguments, describing the leading singularities of the physical quantities, e.g., of the magnetic susceptibility:

$$
\chi(\tau) \sim \Gamma_{ \pm}|\tau|^{-\gamma}, \quad \gamma=\nu\left(2-\eta_{\phi}\right) .
$$

In the non-asymptotic regime, the system approaches criticality in a more complex way and this is where corrections to scaling appear,

$$
\chi(\tau) \sim \Gamma_{ \pm}|\tau|^{-\gamma}\left(1+\Gamma_{ \pm}^{\prime}|\tau|^{\nu \omega}+\Gamma^{\prime \prime}{ }_{ \pm}|\tau|^{2 \nu \omega}+\cdots\right),
$$

where the scaling dimension $\omega$ corresponds to the negative of the leading irrelevant RG eigenvalue, $\omega=-\left|y_{3}\right|$, as it is usually denoted, and the dots in equation (12) stand for higher order irrelevant corrections. Non solum the critical exponents, sed etiam combinations of critical amplitudes and correction-to-scaling exponents are universal quantities.

Also it is common practice, in order to describe the approach to criticality especially in experiments and simulations, to introduce effective exponents through

$$
\chi(\tau) \sim \Gamma_{ \pm}|\tau|^{-\gamma_{\mathrm{eff}}}, \quad \gamma_{\mathrm{eff}}=-\frac{\mathrm{d} \ln \chi(\tau)}{\mathrm{d} \ln |\tau|}
$$




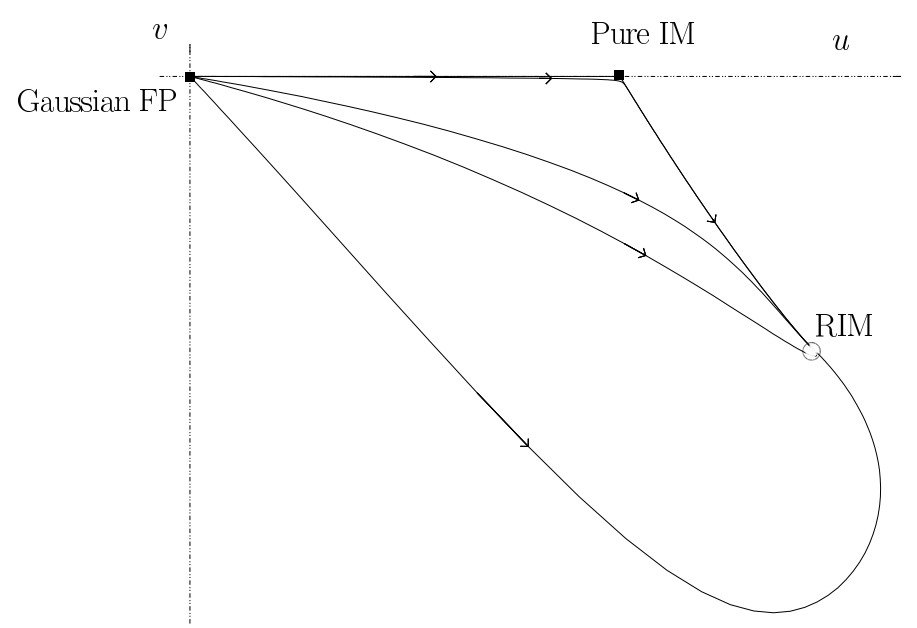

Figure 1. RG flows in the $(u, v)$ plane. The points denoted "Gaussian FP", "Pure IM" and "RIM" are respectively the Gaussian fixed point, the pure Ising model fixed point and the random Ising model fixed point.

These effective exponents may be calculated theoretically from the flow equations, e.g.,

$$
\begin{aligned}
\eta_{\mathrm{eff}}(l) & =\gamma_{\phi}(u(l), v(l)), \\
\frac{1}{\nu_{\mathrm{eff}}(l)} & =2-\gamma_{m^{2}}(u(l), v(l)) .
\end{aligned}
$$

The variation of effective exponents depends on the RG flow in the parameter space as shown in figure 1.

In the case of Heisenberg-like ferromagnets, the experimental observation of a maximum of the effective exponent $\gamma_{\text {eff }}$ found a theoretical explanation in terms of trajectories in the parameter space [9]. The same observation holds in the case of the random Ising model, but the critical exponents also change at the disorder fixed point in this latter case.

\section{Experiments}

Experiments on site-diluted three-dimensional Ising magnets are usually performed on uniaxial disordered anti-ferromagnets such as $\mathrm{Fe}_{1-x} \mathrm{Zn}_{x} \mathrm{~F}_{2}$ or $\mathrm{Mn}_{1-x} \mathrm{Zn}_{x} \mathrm{~F}_{2}$. The original aim was the study of the random-field behaviour when a uniform magnetic field is applied to such a disordered system. However, when the samples are of high quality (low mosaicity, high chemical homogeneity), also the behaviour in zero external magnetic field is accessible ( $3 D$ disordered Ising model universality class). Staggered susceptibility and correlation length are deduced from neutron scattering experiments. The scattering intensity $I(\mathbf{q})$ is the Fourier transform of the pair correlation function, where long-range fluctuations produce an isotropic Lorentzian peak centred at the superstructure spot position $\mathbf{q}_{0}$ with a peak intensity given by 
the susceptibility and a width determined by the inverse correlation length, while long-range order gives a background proportional to the order parameter squared:

$$
I(\mathbf{q})=\left\langle m^{2}\right\rangle \delta\left(\mathbf{q}-\mathbf{q}_{0}\right)+\frac{\chi}{1+\xi^{2}\left(\mathbf{q}-\mathbf{q}_{0}\right)^{2}} .
$$

Fitting the Lorentzian at different temperatures eventually give access to the critical exponents, critical amplitudes, and possibly the correction to scaling.

\section{Monte Carlo simulations}

The majority of numerical studies of the disordered Ising model were concerned with site dilution. But we may also choose to model the disorder by bond dilution in order to compare these two kinds of disorder and to verify that they indeed lead to the same set of new critical exponents, as expected theoretically by universality. In our study we therefore considered the bond-diluted Ising model in three dimensions whose Hamiltonian with uncorrelated quenched random interactions can be written (in a Potts model normalization) as

$$
-\beta \mathcal{H}=\sum_{(i, j)} K_{i j} \delta_{\sigma_{i}, \sigma_{j}}
$$

where the spins take the values $\sigma_{i}= \pm 1$ and the sum goes over all nearest-neighbour pairs $(i, j)$. The coupling strengths are allowed to take two different values $K_{i j}=$ $K \equiv J / k_{\mathrm{B}} T$ and 0 with probabilities $p$ and $1-p$, respectively,

$$
\mathcal{P}\left[K_{i j}\right]=\prod_{(i, j)} P\left(K_{i j}\right)=\prod_{(i, j)}\left[p \delta\left(K_{i j}-K\right)+(1-p) \delta\left(K_{i j}\right)\right]
$$

$c=1-p$ being the concentration of the missing bonds, which play the role of non-magnetic impurities.

The phase diagram and the critical properties at a few selected dilutions were studied by large-scale Monte Carlo simulations on simple cubic lattices with $V=L^{3}$ spins (up to $L=96$ ) and periodic boundary conditions in the three space directions, using the Swendsen-Wang cluster algorithm for updating the spins. All physical quantities are averaged over 2000-5000 disorder realizations, indicated by a bar (e.g., $\bar{\chi}$ for the susceptibility). Standard definitions were used, e.g., for a given disorder realization, the magnetization is defined according to $m=\langle|\mu|\rangle$ where $\langle\cdots\rangle$ stands for the thermal average and $\mu=\left(N_{\uparrow}-N_{\downarrow}\right) /\left(N_{\uparrow}+N_{\downarrow}\right)$ with $N_{\uparrow, \downarrow}$ counting the number of "up" and "down" spins. The susceptibility follows from the fluctuationdissipation relation, $\chi=K V\left(\left\langle\mu^{2}\right\rangle-\langle|\mu|\rangle^{2}\right)$. The phase diagram is obtained by locating the maxima of the average susceptibility $\bar{\chi}_{L}$ (a diverging quantity in the thermodynamic limit) for increasing lattice sizes $L$ as a function of the coupling strength $K$.

As a function of the reduced temperature $\tau=\left(K_{\mathrm{c}}-K\right)(\tau<0$ in the lowtemperature (LT) phase and $\tau>0$ in the high-temperature (HT) phase) and the 


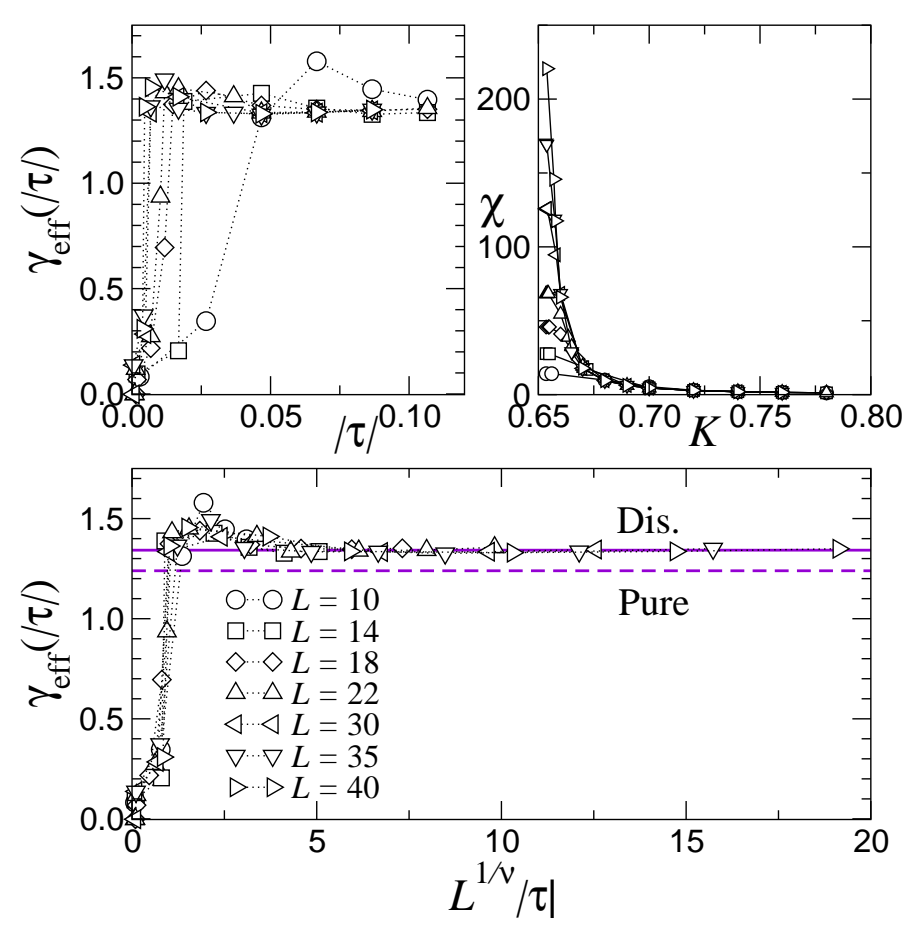

Figure 2. Variation of the temperature dependent effective critical exponent $\gamma_{\text {eff }}(|\tau|)=-\mathrm{d} \ln \bar{\chi} / \mathrm{d} \ln |\tau|$ (in the low-temperature phase) as a function of the reduced temperature $|\tau|$ (top) and $L^{1 / \nu}|\tau|$ (bottom) for the bond-diluted Ising model with $p=0.7$ and several lattice sizes $L$. The horizontal solid and dashed lines indicate the site-diluted and pure values of $\gamma$, respectively.

system size $L$, the susceptibility is expected to scale as:

$$
\bar{\chi}(\tau, L) \sim|\tau|^{-\gamma} g_{ \pm}\left(L^{1 / \nu}|\tau|\right)
$$

where $g_{ \pm}$is a scaling function of the variable $x=L^{1 / \nu}|\tau|$ and the subscript \pm stands for the HT/LT phases. Recalling (13) we can define a temperature dependent effective critical exponent $\gamma_{\text {eff }}(|\tau|)=-\mathrm{d} \ln \bar{\chi} / \mathrm{d} \ln |\tau|$, which should converge towards the asymptotic critical exponent $\gamma$ when $L \rightarrow \infty$ and $|\tau| \rightarrow 0$. Our results for $p=0.7$ are shown in figure 2.

For the greatest sizes, the effective exponent $\gamma_{\text {eff }}(|\tau|)$ is stable around 1.34 when $|\tau|$ is not too small, i.e., when the finite-size effects are not too strong. The plot of $\gamma_{\text {eff }}(|\tau|)$ vs. the rescaled variable $L^{1 / \nu}|\tau|$ shows that the critical power-law behaviour holds in different temperature ranges for the different sizes studied. From the temperature behaviour of the susceptibility, we also have directly extracted the power-law exponent $\gamma$ using error weighted least-squares fits by choosing the temperature range that gives the smallest $\chi^{2} /$ d.o.f for several system sizes. The results are consistent with $\gamma \approx 1.34-1.36$.

From the previous expression of the susceptibility as a function of the reduced temperature and size, it is instructive to plot the scaling function $g_{ \pm}(x)$. For finite size and $|\tau| \neq 0$, the scaling functions may be Taylor expanded in powers of the 


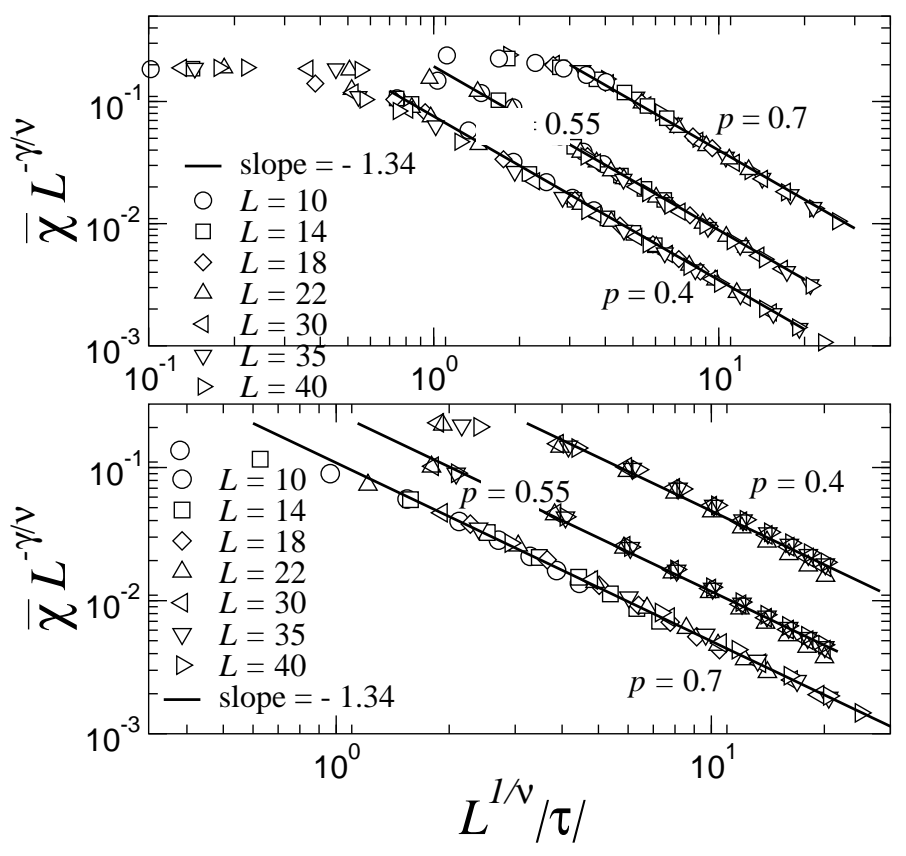

Figure 3. $L o g-\log$ plot of the scaling function $\bar{\chi} L^{-\gamma / \nu}$ in the LT and HT phases (respectively top and bottom) against $L^{1 / \nu}|\tau|$ for $p=0.4,0.55$, and 0.7 . The solid lines show the power-law behaviours with the exponent $\gamma \simeq 1.34$ which characterise the disordered fixed point. The data for the smallest values of $L^{1 / \nu}|\tau|$, which do not lie on the master curve, illustrate the finite-size effects when the correlation length $\xi$ is limited by the linear size $L$.

inverse scaling variable $x^{-1}=\left(L^{1 / \nu}|\tau|\right)^{-1}, \bar{\chi}_{ \pm}(\tau, L)=|\tau|^{-\gamma}\left[g_{ \pm}(\infty)+x^{-1} g_{ \pm}^{\prime}(\infty)+\right.$ $O\left(x^{-2}\right)$ ], where the amplitude $g_{ \pm}(\infty)$ is usually denoted by $\Gamma_{ \pm}$. Multiplying by $L^{-\gamma / \nu}$ leads to

$$
\bar{\chi}_{ \pm} L^{-\gamma / \nu}=x^{-\gamma} g_{ \pm}(x)=\Gamma_{ \pm} x^{-\gamma}+O\left(x^{-\gamma-1}\right) .
$$

The curves in the ordered and disordered phases, shown in figure 3, are obviously universal master curves whose slopes, in a log-log plot, give the critical exponent $\gamma \simeq 1.34$. Indeed, when $|\tau| \rightarrow 0$ but with $L$ still larger than the correlation length $\xi$, one should recover the critical behaviour given by $g_{ \pm}(x)=O(1)$. The critical amplitudes $\Gamma_{ \pm}$follow.

\section{Results and conclusions}

In conclusion, according to Sagredo's suggestion we may compare the results deduced from the different techniques. We only concentrate here on the behaviour of the susceptibility, which already leads to partially conflicting results as can be seen by inspection of table 1 . How to conclude in favour of universality? A possible answer would be as follows. 
In the same pub, a few days later, after inspection of the results:

SAGR. Universality is still a good idea, but it is very difficult to produce high-quality samples where universality is clearly satisfied.

Simpl. For the simulations, it is so time consuming to increase the size of the system that it is not feasible at the moment. The problems here come essentially from the thermodynamic limit and the disorder average.

SALV. In the equations, the sample is perfect, the disorder average is exact and the thermodynamic limit is automatically understood. So I believe that theoretical results are correct.

SimPL. AND SAGR. But what about $R G$ calculations at 7 loop approximation? Will it come from St-Petersburg, from Roma, or from the Linz-Lviv axis?

Table 1. Critical exponents and critical amplitude ratio of the susceptibility as measured with different techniques.

\begin{tabular}{|l|l|l|l|l|}
\hline Technique & $\gamma$ & $\Gamma_{+} / \Gamma_{-}$ & $\omega$ & Ref. \\
\hline RG & & 2.2 & & {$[10]$} \\
& 1.318 & & $0.39(4)$ & {$[11,12]^{1}$} \\
& $1.330(17)$ & & $0.25(10)$ & {$[4]^{2}$} \\
\hline Neutron scattering & $1.44(6)$ & 2.2 & 0.5 & {$[5]^{3}$} \\
& $1.31(3)$ & $2.8(2)$ & & {$[13]^{4}$} \\
& $1.37(4)$ & $2.40(2)$ & & {$[14]^{5}$} \\
\hline MC & $1.342(10)$ & & 0.37 & {$[15]^{6}$} \\
& $1.34(1)$ & $1.62(10)$ & undetermined & {$[16]^{7}$} \\
& $1.342(7)$ & & undetermined & {$[17]^{8}$} \\
\hline
\end{tabular}

14 loop approximation.

${ }^{2} 6$ loop approximation, fixed dimension.

${ }^{3} \mathrm{Fe}_{1-x} \mathrm{Zn}_{x} \mathrm{~F}_{2}, x=0.4,0.5,|\tau| \sim 10^{-2}$.

${ }^{4} \mathrm{Fe}_{0.46} \mathrm{Zn}_{0.54} \mathrm{~F}_{2}, 1.5 \times 10^{-3} \leqslant|\tau| \leqslant 10^{-1}$.

${ }^{5} \mathrm{Mn}_{0.75} \mathrm{Zn}_{0.25} \mathrm{~F}_{2}, 4 \times 10^{-4} \leqslant|\tau| \leqslant 2 \times 10^{-1}$.

${ }^{6}$ site dilution, $p=0.4$ to 0.8 .

7 bond dilution, $p=0.7$. The correction to scaling is too small to be determined.

8 site dilution, $p=0.8$. The observed correction to scaling could be the next-to-leading.

\section{Acknowledgements}

It is a great pleasure to thank Yurko Holovatch who gave us the opportunity to contribute to the Festschrift dedicated to the 60th birthday of Reinhard Folk. 


\section{References}

1. Khmel'nitskiǔ D.E., Sov. Phys. JETP, 1974, 41, 981.

2. Harris A.B., J. Phys. C, 1974, 7, 1671.

3. Folk R., Holovatch Yu., Yavors'kii T., Physics-Uspekhi, 2003, 46, 169.

4. Pelissetto A., Vicari E., Phys. Rev. B, 2000, 62, 6393.

5. Birgeneau R.J., Cowley R.A., Shirane G., Yoshizawa H., Belanger D.P., King A.R., Jaccarino V., Phys. Rev. B, 1983, 27, 6747.

6. Landau D.P., Phys. Rev. B, 1980, 22, 2450.

7. Fermi E., Pasta J., Ulam S. Collected Papers of Enrico Fermi, ed. Segré E. University of Chicago Press, Chicago, 1955.

8. Holovatch Yu., Blavats'ka V., Dudka M., von Ferber C., Folk R., Yavors'kii T., Int. J. Mod. Phys. B, 2002, 16, 4027.

9. Dudka M., Folk R., Holovatch Yu., Ivaneiko D., J. Magn. Magn. Mat., 2003, 256, 243.

10. Newlove S.A., J. Phys. C: Solid State Phys., 1983, 16, L423.

11. Folk R., Holovatch Yu., Yavors'kii T., J. Phys. Stud., 1998, 2, 213.

12. Folk R., Holovatch Yu., Yavors'kii T., Phys. Rev. B, 2000, 61, 15114.

13. Belanger D.P., King A.R., Jaccarino V., Phys. Rev. B, 1986, 34, 452.

14. Mitchell P.W., Cowley R.A., Yoshizawa H., Böni P., Uemura Y.J., Birgeneau R.J., Phys. Rev. B, 1986, 34, 4719.

15. Ballesteros H.G., Fernández L.A., Martín-Mayor V., Muñoz-Sudupe A., Parisi G., Ruiz-Lorenzo J.J., Phys. Rev. B, 1998, 58, 2740.

16. Berche P.E., Chatelain C., Berche B., Janke W., Eur. Phys. J. B, 2004, 39, 463.

17. Calabrese P., Martín-Mayor V., Pelissetto A., Vicari E., Phys. Rev. E, 2003, 68, 036136. 
Тривимірна випадкова модель Ізинга: теорія, експеримент та симуляції - складне співіснування

\author{
Б.Берш ${ }^{1}$, П.І.Берш ${ }^{2}$, К.Шатлен ${ }^{1}$, В.Янке ${ }^{3}$
}

1 Група М, Лабораторія фізики матеріалів, УМР СНPC 7556, Університет Анрі Пуанкаре, Нансі 1,

Ф-54506 Вандувр лє Нансі Седекс, Франція

2 Група фізики матеріалів, УМР СНРC 6634, Університет міста Руен,

Ф-76801 Сан Етьєн де Руврей Седекс, Франція

3 Інститут теоретичної фізики,

Університет міста Ляйпціг,

D-04109 Ляйпціг, Німеччина

Отримано 11 листопада 2004 р.

Ми обговорюємо різні підходи до вивчення впливу безладу в тривимірній моделі Ізинга. 3 теоретичної точки зору, обчислення за допомогою ренормалізаційної групи забезпечують досить добрі результати. Експерименти, виконані на кристалічних сумішах компонентів дають значення критичних показників з точністю до трьох значень після коми. Чисельно, великі Монте Карло симуляції також претендують на співмірну точність. Задача стає складнішою коли порівнювити деталі трьох підходів.

Ключові слова: випадкова модель Ізинга, ренормалізаційна група, Монте Карло симуляції, ефективні критичні показники

PACS: 05.40.+j, 64.60.Fr, 75.10.Hk 